\title{
Fatores que afetam os índices antropométricos infantis: um enfoque nas famílias monoparentais femininas
}

\author{
Factors that influence children's anthropometric indices: \\ a focus on female single-parent families
}

Taytiellen Fernandes Alves (https://orcid.org/0000-0002-2465-1356) ${ }^{1}$

Alexandre Bragança Coelho (https://orcid.org/0000-0002-9735-7035) ${ }^{1}$

Guilherme Fonseca Travassos (https://orcid.org/0000-0002-9868-7386) ${ }^{1}$

${ }^{1}$ Universidade Federal de Viçosa. Av. Peter Henry Rolfs s/n, Campus Universitário. 36570900 Viçosa MG Brasil. taytiellen@hotmail.com

\begin{abstract}
In the last decades several alterations have occurred in the dynamics of the organization of families, including changes in size, structure, and composition. Among new family arrangements, the increase in female single-parent families stands out. This structure tends to be in a situation of greater social vulnerability in relation to other arrangements. With this in mind, the scope of this study sought to analyze the relationship between the female single-parent family arrangement and anthropometric measurements of under five-year-old offspring, with data from the 2008-2009 Brazilian household budget survey (POF/IBGE). Two equations for the analysis of anthropometric measurements, with the $z$-score of "height-for-age" and the z-score of "weight-for -height" as dependent variables, were estimated. The results revealed that, taking other important variables into consideration, such as income, education and domestic characteristics pertaining to the "female single-parent" arrangement, had a positive effect on anthropometric measurements when compared with the "couple with children" arrangement, indicating that in households in which the mother does not have a spouse in residence, children had better long-term health indicators than in households in which the spouse was present.
\end{abstract}

Key words Single-parent family, Nutritional status, Children
Resumo Nas últimas décadas aconteceram várias alterações no padrão de organização das famílias, como mudanças no tamanho, estrutura e composição. Dentre os novos arranjos familiares, destaca-se o crescimento de famílias monoparentais femininas. Este arranjo tende a se encontrar em situação de maior vulnerabilidade social em relação a outros arranjos. Diante disso, este estudo buscou analisar a relação entre o arranjo monoparental feminino e o estado nutricional de crianças menores de cinco anos, com dados da Pesquisa de Orçamentos Familiares (POF/IBGE) de 20082009. Para isso, estimaram-se duas equações para análise de indices antropométricos, tendo como variáveis dependentes o escore $z$ da "altura para idade" e o escore $z$ do "peso para altura". Os resultados mostraram que, controlando para outras variáveis importantes como renda, escolaridade e características domiciliares, pertencer ao arranjo "monoparental feminino" teve efeito positivo sobre o escore $z$ da "altura para idade" quando comparado ao arranjo "casal com filhos", indicando que a presença da mãe, sem o cônjuge, contribui para melhorar esse indicador de saúde de longo prazo.

Palavras-chave Família monoparental, Estado nutricional, Crianças 


\section{Introdução}

A família é um dos eixos responsáveis pela qualidade de vida de seus componentes e onde ocorrem várias decisões referentes à moradia, saúde, alimentação e educação ${ }^{1}$. Houve nos últimos anos muitas mudanças na composição familiar, devido a transformações econômicas, sociais e sobretudo demográficas, e aconteceram também várias alterações no padrão de organização das famílias, como mudanças no tamanho, estrutura e composição. Assim, ainda que o modelo tradicional de família prevaleça, reduziu-se sua importância ao aumentar a participação de outros arranjos familiares, constituídos principalmente por casal sem filhos, unipessoais e monoparentais $^{1,2}$.

Dentre os novos arranjos familiares, destaca-se o crescimento de famílias monoparentais femininas. No Brasil, em 2001, esse arranjo totalizava 9 milhões de famílias, passando para 11,6 milhões em 2015, um crescimento de $28,3 \%$ no período $^{3}$. Em termos percentuais, esse arranjo representava cerca de $15,3 \%$ das famílias em 2010 , contra $11,5 \%$ em $1980^{4}$. As causas desse crescimento estão ligadas a uma série de questões, como novos valores culturais e comportamentais, que levam ao aumento de separações e divórcios e maior independência feminina ${ }^{3}$.

Por outro lado, o arranjo familiar monoparental feminino tende a apresentar maior incidência de pobreza e insegurança alimentar. De acordo com Maia et al. ${ }^{5}$, a renda per capita da família monoparental feminina era de $\mathrm{R} \$ 450,00$ em 2012, contra R\$653,00 da monoparental masculina. Esse baixo nível de renda se reflete nos índices de pobreza: em 2012, o número de famílias monoparentais femininas classificadas como pobres foi de 1,8 milhões, ou seja, $16,30 \%$ das famílias nesse arranjo foram consideradas pobres $^{5,6}$. Esse quadro aumenta a representatividade desse arranjo nos programas sociais do governo brasileiro. Em 2013, por exemplo, as famílias monoparentais femininas tiveram a maior participação no programa Bolsa-Família (42,2\%), seguida pelo arranjo casal com filhos $(37,6 \%)^{7}$.

Além disso, as mudanças ocorridas nas últimas décadas na estrutura familiar influenciam o padrão alimentar, tornando-se menos comum as refeições realizadas no domicílio e maior o consumo de alimentos industrializados ${ }^{8}$. Nesse contexto, os índices antropométricos apresentam-se como o método mais utilizado para avaliação do estado nutricional infantil. Fatores relacionados a escolaridade dos pais, idade da criança, altura dos pais, assistência à saúde, acesso ao saneamento básico, condições de moradia, renda familiar e outros são importantes indicadores que influenciam as medidas antropométricas das crianças ${ }^{9,10}$.

O déficit estatural é uma medida de desnutrição em longo prazo e geralmente está associado a uma alimentação inadequada ${ }^{11}$. A desnutrição e a obesidade estão relacionadas às mudanças no estilo de vida e padrões alimentares da população, podendo acarretar sérios problemas, como alta prevalência de doenças crônicas, sendo o padrão de saúde infantil refletido principalmente por meio de medidas antropométricas ${ }^{12}$. No longo prazo, a deficiência nutricional pode provocar atrasos no crescimento das crianças, detectados através da relação "altura x idade". Portanto, analisar essas questões nos arranjos monoparentais femininos pode contribuir na criação de políticas públicas voltadas para a melhoria do estado nutricional infantil, principalmente das famílias de baixa renda ${ }^{13,14}$.

$\mathrm{Na}$ literatura alguns estudos analisaram o perfil antropométrico de crianças e tiveram como resultado a prevalência de déficit de estatura entre as crianças, o que torna importante o acompanhamento nutricional juntamente com fatores socioeconômicos ${ }^{9,11}$.

Nesse contexto, o objetivo desse trabalho foi analisar a relação entre famílias monoparentais femininas e os índices antropométricos das crianças menores de cinco anos. Os índices antropométricos utilizados nesse trabalho são o escore z do "peso para a altura" e o escore z da "altura para idade", que serão explicados mais detalhadamente na seção seguinte. Diante dos fatos apresentados, torna-se importante analisar o desenvolvimento das crianças desse arranjo familiar, visto que pouco se sabe na literatura sobre o efeito desse arranjo familiar no crescimento e no estado nutricional das crianças.

\section{Estratégia empírica}

Para a análise do efeito do arranjo monoparental feminino nos índices antropométricos, foi utilizado o modelo de equações aparentemente não relacionadas (SUR) ${ }^{15}$, que leva em consideração as possíveis correlações existentes entre os erros das diversas equações de um sistema através do estimador de mínimos quadrados generalizados (MQG). Assim, utiliza-se o SUR, pois pressupõe-se que os erros das equações de medidas antropométricas sejam correlacionados, já que os fatores não-sistemáticos incluídos no termo de erro que podem influenciar o escore $\mathrm{z}$ do "peso 
para a altura" devem ser os mesmos que influenciam o escore z da "altura para idade". Dentre esses fatores, pode-se destacar questões de saúde pública (doenças como dengue, por exemplo), de oferta de alimentos (secas, enchentes, etc...), políticas governamentais (distribuição de alimentos, etc...), entre outras.

Dessa forma, o SUR pode ser escrito como:

$$
y_{i}=X_{i} \beta_{i}+\varepsilon_{i}, i=1, \ldots, \mathrm{M}
$$

Assume-se neste modelo que:

i) $\varepsilon_{i}$ é estritamente exógeno a $X_{i}$,

$$
E\left[\varepsilon \mid X_{1}, X_{2}, \ldots, X_{M}\right]=0
$$

ii) a variância é constante em cada equação, porém difere entre as equações,

$$
E\left[\varepsilon_{\mathrm{m}} \varepsilon_{\mathrm{m}}^{\prime} \mid X_{1}, X_{2}, \ldots, X_{M}\right]=\sigma_{m m} I_{T}
$$

iii) os erros são correlacionados entre as equações,

$$
E\left[\varepsilon_{i t} \varepsilon_{j s}^{\prime} \mid X_{1}, X_{2}, \ldots, X_{M}\right]=\sigma_{i j} \text { se } t=s
$$

iv) os erros não estão correlacionados entre as observações,

$$
E\left[\varepsilon_{i t} \varepsilon_{j s}^{\prime} \mid X_{1}, X_{2}, \ldots, X_{M}\right]=0 \text { se } t \neq s
$$

O estimador de MQG de $\beta$ quando a matriz de covariância $(\Omega)$ é conhecido é:

$$
\begin{aligned}
\hat{\beta}_{\mathrm{MQG}}= & {\left[\mathrm{X}^{\prime} \Omega^{-1} \mathrm{X}\right]^{-1} \mathrm{X}^{\prime} \Omega^{-1} \mathrm{Y}=} \\
& {\left.\left[\mathrm{X}^{\prime}\left(\Sigma^{-1} \otimes \mathrm{I}\right) \mathrm{X}\right]^{-1} \mathrm{X}^{\prime}\left(\Sigma^{-1} \otimes \mathrm{I}\right) \mathrm{Y}\right], }
\end{aligned}
$$

Em que $\hat{\beta}_{\mathrm{MQG}}$ é o estimador MQG de $\beta$; X é a matriz de variáveis explicativas; $Y$ é a matriz de variáveis dependentes; $\Omega$ é a matriz de covariância $\left(\sum \otimes \mathrm{I}\right)$, sendo $\otimes$ o produto kronecker e I a matriz identidade.

Por outro lado, quando a matriz de covariância não é conhecida, utiliza-se o MQF (Mínimos Quadrados Factíveis), feito em dois estágios de estimação. No primeiro estágio, cada equação é estimada por MQO e os resíduos das M equações são usados para estimar $\Omega$, já no segundo, substitui-se $\hat{\Omega}$ para cada $\Omega$ do estimador MQG:

$$
\hat{\beta}_{\mathrm{MQF}}=\left[\mathrm{X}^{\prime}\left(\sum^{-1} \otimes \mathrm{I}\right) \mathrm{X}\right]^{-1} \mathrm{X}^{\prime}\left(\sum^{-1} \otimes \mathrm{I}\right) \mathrm{Y},
$$

Em que $\hat{\beta}_{\mathrm{MQF}}$ é o estimador MQF de $\beta$; X é a matriz de variáveis explicativas; $Y$ é a matriz de variáveis dependentes; ' $\left(\Sigma^{-1} \otimes \mathrm{I}\right)$ é a matriz de covariância estimada a partir dos resíduos do primeiro estágio.

A ideia foi estimar duas equações para dois dos principais índices antropométricos (escore $\mathrm{z}$ do "peso para altura" e escore $\mathrm{z}$ da "altura para idade") medindo o efeito de variáveis de tipo de arranjo domiciliar sobre esses índices para crianças de zero a cinco anos. Uma série de variáveis de controle foram também adicionadas, englobando variáveis de localização domiciliar, características das crianças, características domiciliares, características locacionais e características de alimentação. Interações entre tipo de arranjo familiar e qualidade da alimentação também foram utilizadas.

Os dados utilizados foram provenientes dos microdados da Pesquisa de Orçamentos Familiares (POF) 2008-200916. Essa pesquisa é de caráter amostral de 55.970 domicílios, realizada pelo Instituto Brasileiro de Geografia e Estatística $(\text { IBGE })^{17}$. Das 14.866 observações referentes à domicílios com crianças até cinco anos de idade, 1.975 observações não informaram alguma das variáveis demográficas consideradas e foram excluídas da análise. Além disso, 3.741 observações foram retiradas da amostra, pois eram referentes à domicílios que não consumiram nenhum dos alimentos. Dessa forma, a amostra final possui 9.150 observações. Além da variável “arranjo monoparental feminino", outras variáveis importantes foram usadas como controle para a análise. Essas variáveis estão descritas no Quadro 1. Ressalta-se que "Urbano", "Região Sudeste", "Filhos de 4 a 5 anos" e "Casal com filhos" são as categorias base.

Assim, as formas funcionais utilizadas para analisar as medidas antropométricas foram baseadas em Haddad e Hoddinott ${ }^{10}$ : 
Quadro 1. Variáveis selecionadas para análise das medidas antropométricas.

\begin{tabular}{|c|}
\hline Variáveis Dependentes \\
\hline $\mathrm{PA}=$ escore $\mathrm{z}$ do "peso para altura" \\
\hline $\mathrm{AI}=$ escore $\mathrm{z}$ da "altura para idade" \\
\hline Variáveis Explicativas \\
\hline Localização domiciliar \\
\hline Rural $=$ Domicílio localizado na zona rural $=1 ;$ caso contrário $=0$ \\
\hline Norte $=$ Domicílio localizado na região Norte $=1$; caso contrário $=0$ \\
\hline Nordeste $=$ Domicílio localizado na região Nordeste $=1 ;$ caso contrário $=0$ \\
\hline Sul = Domicílio localizado na região Sul $=1 ;$ caso contrário $=0$ \\
\hline Centro-Oeste $=$ Domicílio localizado na região Centro-Oeste $=1$; caso contrário $=0$ \\
\hline Características das crianças \\
\hline Sexofilho $=$ Filho do sexo feminino $=1$, Caso contrário $=0$ \\
\hline Primogênito $=$ Filho primogênito $=1$, Caso contrário $=0$ \\
\hline Filhos $0-1=$ Presença de filho $(\mathrm{s})$ com idade entre 0 e 1 ano $=1$; caso contrário $=0$ \\
\hline Filhos $1-2=$ Presença de filho(s) com idade entre 1 e 2 anos $=1$; caso contrário $=0$ \\
\hline Filhos2-3 = Presença de filho(s) com idade entre 2 e 3 anos $=1$; caso contrário $=0$ \\
\hline Filhos3-4 = Presença de filho(s) com idade entre 3 e 4 anos $=1$; caso contrário $=0$ \\
\hline Arranjo familiar \\
\hline $\begin{array}{l}\text { Monoparentalfemin }=\text { Domicílio composto por responsável do sexo feminino e com pelo menos um filho(a) } \\
=1 ; \text { Caso contrário }=0\end{array}$ \\
\hline $\begin{array}{l}\text { Monoparentalmasc }=\text { Domicílio composto por responsável do sexo masculino e com pelo menos um } \\
\text { filho }(\mathrm{a})=1 \text {; Caso contrário }=0\end{array}$ \\
\hline $\begin{array}{l}\text { Outrosarranjos }=\text { Outros tipos de domicílios constituídos de forma distinta das anteriores }=1 ; \text { caso contrário } \\
=0\end{array}$ \\
\hline Características domiciliares \\
\hline Escolresponsável $=$ Anos de estudo do responsável pelo domicílio \\
\hline Renda $=$ Renda mensal per capita \\
\hline Idaderesponsável $=$ Idade do responsável pelo domicílio \\
\hline Alturaresponsável $=$ Altura do responsável pelo domicílio \\
\hline Características locacionais \\
\hline Águacanalizada $=$ Domicílio com água canalizada $=1 ;$ Caso contrário $=0$ \\
\hline Proxesgoto $=$ Domicílio próximo a esgoto a céu aberto ou valão $=1 ;$ Caso contrário $=0$ \\
\hline Escoadsanitário $=$ Existência de rede coletora de esgoto $=1 ;$ Caso contrário $=0$ \\
\hline $\begin{array}{l}\text { Materialparedes }=\text { Material que predomina nas paredes externas do domicílio é Alvenaria }=1 \text {; Caso contrário } \\
=0\end{array}$ \\
\hline Materialcobertura $=$ Material que predomina na cobertura do domicílio é telha $=1 ;$ Caso contrário $=0$ \\
\hline Característica da alimentação \\
\hline $\begin{array}{l}\text { Qualidade da alimentação = Variável proxy para qualidade da alimentação, criada baseada na demanda } \\
\text { domiciliar dos alimentos: alimentos preparados, refrigerantes, frutas e hortaliças. Se o indivíduo tem má } \\
\text { alimentação }=1 \text {; Caso contrário }=0\end{array}$ \\
\hline Quantidadealimento $=$ Quantidade de alimento consumido é sempre suficiente $=1$; Caso contrário $=0$ \\
\hline Interações entre tipo de arranjo familiar e qualidade da alimentação \\
\hline $\begin{array}{l}\text { MonoparentalfeminQualidadealimentação = variável que capta o efeito de interação entre o arranjo } \\
\text { monoparental feminino e a qualidade da alimentação }\end{array}$ \\
\hline $\begin{array}{l}\text { MonoparentalmascQualidadealimentação = variável que capta o efeito de interação entre o arranjo } \\
\text { monoparental masculino e a qualidade da alimentação }\end{array}$ \\
\hline $\begin{array}{l}\text { OutrosarranjosQualidadealimentação = variável que capta o efeito de interação entre outros arranjos e a } \\
\text { qualidade da alimentação }\end{array}$ \\
\hline
\end{tabular}

Fonte: Elaborado pelos autores a partir das informações da POF (2008-2009). 
$\mathrm{PA}_{\mathrm{ik}}\left(\right.$ ou $\left.\mathrm{AI}_{\mathrm{ik}}\right)=\beta_{0}+\beta_{1}$ Monoparentalfemin $_{k}+$ $\beta_{2}$ Monoparentalmasc $_{k}+\beta_{3}$ Outrosarranjos $_{k}+$ $\beta_{4}$ Escolresponsável $_{k}+\beta_{5}$ Renda $_{k}+\beta_{6}$ Idaderesponsável $_{k}+\beta_{7}$ Alturaresponsável $_{k}+\beta_{8}$ Águacanalizada ${ }_{k}+\beta_{39}$ Proxesgoto $_{k}+\beta_{10}$ Escoadsanitário $_{k}$ $+\beta_{11}$ Materialparedes $_{k}+\beta_{12}$ Materialcobertura $_{k}$ $+\beta_{13}$ Máalimentação $o_{k}+\beta_{14}$ Quantidadealimento $+\beta_{15}$ Sexofilho $_{k}+\beta_{16}$ Filhos $0-1_{k}+\beta_{17}$ Filhos1 $2_{k}+$ $\beta_{18}$ Filhos $2-3_{k}+\beta_{10}$ Filhos3-4 ${ }_{k}+\beta_{20}$ MonoparentalfeminMáalimentação $o_{k}+\beta_{21}$ MonoparentalmascMáalimentação ${ }_{k}+\beta_{22}$ OutrosarranjosMáalimentação ${ }_{k}+\beta_{23}$ Primogênito $+\beta_{24}$ Rural $+\beta_{25}$ Norte + $\beta_{26}$ Nordeste $+\beta_{27}$ Sul $+\beta_{28}$ CentroOeste $+\varepsilon_{i k}$,

As variáveis das equações (1.7) estão indexadas por $i$ representando as medidas antropométricas das crianças menores de cinco anos de idade, $k$ representa o domicílio e $\varepsilon_{i k}$ é o termo de erro; $\beta$ 's são os parâmetros a serem estimados.

As variáveis dependentes descritas no Quadro 1 representam o escore $\mathrm{z}$ da "altura para idade" e do "peso para altura", de acordo com o sexo da criança. $O$ escore $z$ é a relação entre o valor observado para o indivíduo (peso ou altura) menos o valor da mediana da população de referência, dividido pelo desvio padrão da população de referência. Para análise do escore $\mathrm{z}$, foi necessário estratificá-los por faixa etária, uma vez que há diferenças na análise do estado nutricional entre crianças de 0 a 2 anos e de idades superiores.

A altura para idade é considerada uma medida que representa o estado de saúde a longo prazo, enquanto o peso para altura representa o estado de saúde a curto prazo, pois esta medida é mais sensível às flutuações de curto prazo. Foram utilizados os valores da mediana, de acordo com o sexo da criança, calculados de acordo com as curvas de crescimento adotadas pela Organização Mundial da Saúde ${ }^{18}$.

Em relação às características das crianças, as variáveis referentes a idade do filho foram incluídas para uma análise mais detalhada da faixa etária sobre as variáveis dependentes. Foram escolhidas crianças de até cinco anos de idade, porque esta faixa etária é de importantes aquisições no processo de desenvolvimento e essas crianças geralmente possuem maior risco nutricional e são mais vulneráveis a outros problemas da infância ${ }^{19}$. Já a variável de filho primogênito analisa, dentre os filhos da amostra, se o fato de ser primogênito influencia de alguma forma os índices antropométricos.

Sobre as variáveis referentes a características domiciliares, a escolaridade e renda foram incluídas, pois acredita-se que filhos de pais com menor escolaridade e renda possuem maior probabilidade de apresentar obesidade e/ou desnutrição e déficit de estatura ${ }^{10,20}$. A idade do responsável foi incluída uma vez que há a hipótese de que pais muito jovens tenderão a ter filhos menores. Por fim, a altura foi incluída para captar o efeito da genética sobre as medidas antropométricas ${ }^{10,21}$.

Em relação as variáveis de características locacionais, elas foram incluídas devido ao fato de que o acesso a serviços de infraestrutura básica é um dos fatores associados às melhorias nas medidas antropométricas'. Sobre às variáveis de localização domiciliar, foram incluídas para captar o efeito dos fatores regionais sobre as medidas antropométricas.

Como a má alimentação pode influenciar o crescimento e desenvolvimento das crianças, a variável Qualidade da alimentação foi inserida no modelo e é baseada nas despesas com alimentação no domicílio: se as despesas no domicílio com alimentos pouco nutritivos (alimentos preparados e refrigerantes) for maior do que as despesas com alimentos de maior valor nutricional (frutas e hortaliças), este domicílio foi caracterizado como tendo má qualidade da alimentação. Ressalta-se que diversos estudos, como o de Souza et al..$^{22}$, consideram as frutas e hortaliças como alimentos saudáveis e alimentos preparados e refrigerantes como alimentos não saudáveis. No caso deste estudo, os alimentos preparados são produtos como batata congelada, farofa para viagem, salgadinho, batata frita para viagem, entre outros, de acordo com a definição da POF 20082009. Já a variável quantidade de alimento referese a uma pergunta inserida na POF em que cada informante relatou se a quantidade de alimento consumida no domicílio é sempre suficiente, às vezes não é suficiente, ou normalmente não é suficiente. Ela foi inserida no modelo para analisar o efeito sobre as medidas antropométricas quando a quantidade de alimento no domicílio é sempre suficiente. As variáveis "Urbano", "Sudeste", "Filhos de 4 a 5 anos", "Casal com filhos" e a interação entre "casal com filho e qualidade da alimentação" são as categorias base.

As variáveis de arranjo familiar foram incluídas para fazer uma análise da influência do arranjo familiar sobre as medidas antropométricas das crianças, uma vez que há a hipótese de que famílias monoparentais femininas tem uma influência negativa sobre as medidas antropométricas infantis. Já as dummies de interação entre arranjo familiar e má alimentação foram incluídas com o intuito de captar se o efeito da má alimentação é diferente por tipo de arranjo familiar sobre as 
medidas antropométricas. Assim, será possível uma melhor análise da má alimentação e o arranjo familiar sobre a altura para idade e peso para altura das crianças.

\section{Resultados e discussão}

\section{Estatísticas descritivas}

Na Tabela 1 encontra-se a porcentagem, para cada arranjo familiar, de crianças que estão fora da mediana do "peso para altura" e "altura para idade". Seguindo a classificação da OMS, considera-se "fora da mediana", para a variável "altura para idade", as crianças de muito baixa altura para idade (escore $\mathrm{z}<-3$ ) e crianças de baixa altura para idade (escore $-3<z<-2$ ). Já para a medida de peso para altura, considera-se "fora da mediana" crianças com escore $\mathrm{z}<-3$ (crianças com magreza acentuada) e com escore $-3<z<-2$ (crianças com magreza) e também crianças com escore $\mathrm{z}>1$, com risco de sobrepeso e obesidade. Observa-se que, no arranjo monoparental masculino, $13,35 \%$ das crianças estão fora da mediana do peso para altura, e na análise da altura para idade observa-se que no arranjo "monoparental masculino" $16,96 \%$ das crianças estão fora da mediana.

Na Tabela 2 encontram-se as médias das variáveis demográficas. Em relação às características das crianças, observa-se que a maior parte da amostra é composta por crianças do sexo feminino $(71,35 \%)$ e, dos filhos incluídos na amostra, $93,21 \%$ são primogênitos. Essa maior quantidade de crianças do sexo feminino foi surpreendente: uma explicação possível pode ser o fato de que, segundo o $\mathrm{IBGE}^{23}$, crianças do sexo masculino são, em geral, mais frágeis a alguns tipos de doenças e, portanto, mais suscetíveis a mortalidade infantil.

Além disso, cerca de 23,34\% dos domicílios possuem crianças entre 4 e 5 anos, seguido por domicílios com crianças entre 3 e 4 anos (20,68\%), 0 e 1 ano (18,33\%), 2 e 3 anos $(18,19 \%)$, e 1 e 2 anos (17,80\%).

Em relação às características domiciliares, tem-se que a idade média do responsável pelo domicílio era de 38,31 anos, com escolaridade média de 6,96 anos, ou seja, não haviam completado o ensino fundamental. A altura do responsável foi de, em média, 167,25 centímetros. Com relação à renda per capita, os domicílios da amostra apresentaram rendimento médio de $\mathrm{R} \$$ 541,42 , valor próximo a um salário mínimo, $\mathrm{R} \$$
Tabela 1. Porcentagem, por arranjo familiar, de crianças que estão fora da mediana das variáveis de medidas antropométricas.

\begin{tabular}{lcc}
\hline \multicolumn{3}{c}{ Medidas antropométricas } \\
\hline \multirow{2}{*}{ Arranjo familiar } & $\begin{array}{c}\text { Escore z } \\
\text { "peso para } \\
\text { altura" }\end{array}$ & $\begin{array}{c}\text { Escore } \mathbf{z} \\
\text { “altura } \\
\text { para } \\
\text { idade” }\end{array}$ \\
\cline { 2 - 3 } & Amostra & $\begin{array}{c}\text { Amostra } \\
\text { (\%) }\end{array}$ \\
\hline Monoparental masculino & 13,35 & 16,96 \\
Monoparental feminino & 11,82 & 11,15 \\
Casal com filhos & 11,41 & 11,18 \\
Outros arranjos & 11,71 & 7,77 \\
\hline
\end{tabular}

Nota: Foi utilizado o peso amostral, denominado na POF como Fator de Expansão 2.

Fonte: Elaborado pelos autores a partir dos resultados da pesquisa.

Tabela 2. Média das variáveis demográficas utilizadas na amostra, 2008-2009.

\begin{tabular}{|c|c|}
\hline & $\begin{array}{c}\text { Amostra } \\
(\%)\end{array}$ \\
\hline \multicolumn{2}{|l|}{ Características das crianças } \\
\hline Sexo do filho & 71,35 \\
\hline Filho Primogênito & 93,21 \\
\hline Domicílios com filhos de 0 a 1 ano & 18,33 \\
\hline Domicílios com filhos de 1 a 2 anos & 17,80 \\
\hline Domicílios com filhos de 2 a 3 anos & 18,19 \\
\hline Domicílios com filhos de 3 a 4 anos & 20,68 \\
\hline Domicílios com filhos de 4 a 5 anos & 23,34 \\
\hline \multicolumn{2}{|l|}{ Características domiciliares } \\
\hline Escolaridade do responsável & 6,96 \\
\hline Renda per capita & 541,42 \\
\hline Idade do responsável & 38,31 \\
\hline Altura do responsável & 167,25 \\
\hline \multicolumn{2}{|l|}{ Características locacionais } \\
\hline Água canalizada & 89,24 \\
\hline Escoadouro sanitário & 95,10 \\
\hline Proximidade ao esgoto & 11,62 \\
\hline Material da parede & 86,02 \\
\hline Material da cobertura & 79,94 \\
\hline \multicolumn{2}{|l|}{ Características da alimentação } \\
\hline Qualidade da alimentação & 34,17 \\
\hline Quantidade de alimento & 53,85 \\
\hline \multicolumn{2}{|c|}{$\begin{array}{l}\text { Nota: Para o cálculo das médias foi utilizado o peso amostral, } \\
\text { denominado na POF como Fator de Expansão } 2 \text {. }\end{array}$} \\
\hline
\end{tabular}


465 , do período de referência da pesquisa (ano de 2009).

Sobre às características da alimentação, $34,17 \%$ dos domicílios apresentavam integrantes com má alimentação e em 53,85\% dos domicílios a quantidade de alimento consumida foi sempre suficiente. Por fim, em relação às características locacionais, $89,24 \%$ dos domicílios possuíam água canalizada e 95,10\% tinham algum tipo de escoadouro sanitário. Além disso, 86,02\% dos domicílios da amostra tinham alvenaria como material das paredes e, em 79,94\%, o material da cobertura do domicílio era telha. Apenas 11,62\% dos domicílios estavam próximos ao esgoto.

Relacionando as características locacionais com o tipo de arranjo familiar, tem-se que, em média, aproximadamente $87 \%$ do arranjo monoparental feminino têm água canalizada, 95\% têm escoadouro sanitário e $16 \%$ estão próximo ao esgoto. Para o arranjo monoparental masculino, esses valores são de, respectivamente, $75 \%$, $90 \%$ e $36 \%$. Já para o arranjo casal com filhos, esses valores são de, respectivamente, $84 \%, 93 \%$ e $11 \%$. Por fim, para outros arranjos, em média aproximadamente $86 \%$ possuem água canalizada, $96 \%$ possuem escoadouro sanitário e $13 \%$ estão próximos ao esgoto. Fica claro assim que os piores indicadores estão relacionados ao arranjo monoparental masculino.

\section{Resultados do modelo de medidas antropométricas}

Os resultados do modelo de medidas antropométricas, que busca analisar, principalmente, o efeito do arranjo monoparental feminino sobre o escore z do "peso para altura" e da "altura para idade" das crianças, são apresentados na Tabela 3. Em relação à localização domiciliar, verificase que, controlando para outras variáveis como renda e escolaridade etc., o fato de o domicílio estar nas regiões Norte e Nordeste, quando comparado com domicílio situado na região Sudeste, afeta negativamente o escore $\mathrm{z}$ da "altura para idade". Assim, se houver dois domicílios com as mesmas características localizados no Sudeste e Nordeste, o que se localiza no Nordeste apresentará crianças com menor escore z da "altura para idade", que é um indicador de saúde de longo prazo. Acredita-se que os domicílios dessas regiões tenham pior cobertura de serviços básicos de saúde, o que pode impactar no crescimento das crianças. Em relação à variável "rural", não foram encontradas diferenças estatisticamente significativas entre dois domicílios iguais loca- lizadas no meio urbano e rural, ou seja, o fato de pertencer ao meio rural não afeta os índices antropométricos.

Examinando agora as características das crianças, o gênero da criança não apresentou influência significativa nos escores z. Na variável de filho primogênito, observa-se que o fato de a criança ser filho primogênito afeta positivamente o escore z do "peso para altura". De acordo com Primo et al. ${ }^{24}$, estudos norte-americanos observaram que a amamentação exclusiva de mães com filho primogênito foi maior do que em filhos que não são primogênitos, pois mães de primeira gestação são mais propensas a amamentar, o que pode influenciar positivamente as medidas antropométricas de filhos primogênitos. Por outro lado, outros estudos divergem dessa afirmação, como é o caso do estudo de Rodrigues et al. ${ }^{20}$.

Passando para a análise da idade das crianças, constata-se que a presença de filhos nas faixas etárias entre 0 e 1 ano afeta positivamente o escore z da "altura para idade" quando comparados a presença de filhos de 4 a 5 anos (categoria padrão). Já a presença de filhos entre 2 e 3 anos e 3 e 4 anos afeta negativamente o escore $z$ da "altura para idade". O escore z da "altura para idade" é uma medida que representa o estado de saúde de longo prazo. Esse resultado mostra que as condições nutricionais parecem se deteriorar com maior intensidade nas faixas a partir de 2 anos, quando a alimentação das crianças passa a ser mais próxima a dos pais e menos dependente do aleitamento materno. Resultados semelhantes foram encontrados Pereira et al. ${ }^{25}$.

Em relação às características domiciliares, destaca-se o efeito positivo da escolaridade do responsável sobre o escore $\mathrm{z}$ da "altura para idade". Como o efeito da renda já está sendo controlado, esse efeito parece indicar uma maior capacidade dos pais em tomar medidas que melhorem à saúde das crianças, como melhores cuidados na saúde e alimentação. Resultados similares foram constatados por Rissin et al. ${ }^{21}$.

No que diz respeito à renda, esta variável apresentou influência positiva e significativa, apesar de pequena, sobre o escore $\mathrm{z}$ da "altura por idade", indicando que mesmo quando controlado para outros fatores, a renda é importante para redução dos problemas da saúde infantil. Resultados análogos foram constatados por Pereira et al. ${ }^{19}$ e Haddad e Hoddinott ${ }^{10}$.

Observa-se também que a idade do responsável pelo domicílio tem influência positiva sobre o escore z da "altura para idade". Muitos estudos sobre saúde infantil indicam que pais mais ve- 
Tabela 3. Resultados do modelo de medidas antropométricas, 2008-2009.

\begin{tabular}{|c|c|c|}
\hline & $\begin{array}{c}\text { Escore } \mathrm{z} \text { da “altura } \\
\text { para idade" }\end{array}$ & $\begin{array}{c}\text { Escore } \mathrm{z} \text { do "peso } \\
\text { para altura" }\end{array}$ \\
\hline \multicolumn{3}{|l|}{ Localização domiciliar } \\
\hline Rural & $-0,029$ & $-0,015$ \\
\hline Norte & $-0,129^{*}$ & $-0,073^{\star * *}$ \\
\hline Nordeste & 0,052 & $-0,040^{\star}$ \\
\hline Sul & 0,110 & 0,048 \\
\hline Centro-Oeste & 0,069 & $-0,035$ \\
\hline \multicolumn{3}{|l|}{ Características das crianças } \\
\hline Sexo do filho & $-0,029$ & $-0,039$ \\
\hline Filho primogênito & $-0,063$ & $0,087^{\star *}$ \\
\hline Filhos $0-1$ & $0,255^{\star * *}$ & $-0,143^{\star \star \star}$ \\
\hline Filhos 1-2 & 0,014 & $-0,062^{* * *}$ \\
\hline Filhos 2-3 & $-0,099^{*}$ & $-0,072^{\star * *}$ \\
\hline Filhos 3-4 & $-0,303^{* * *}$ & $-0,049^{\star *}$ \\
\hline \multicolumn{3}{|l|}{ Arranjo domiciliar } \\
\hline Monoparental feminino & $0,146^{*}$ & 0,015 \\
\hline Monoparental masculino & 0,139 & 0,242 \\
\hline Outros arranjos & $-0,169$ & $-0,007$ \\
\hline \multicolumn{3}{|l|}{ Características domiciliares } \\
\hline Escolaridade do responsável & $0,018^{\star * *}$ & 0,003 \\
\hline Renda & $0,00006^{*}$ & $1,21(10)^{-6}$ \\
\hline Idade do responsável & $0,003^{\star}$ & $-0,0008$ \\
\hline Altura do responsável & $0,019^{* * *}$ & $0,004^{* * *}$ \\
\hline \multicolumn{3}{|l|}{ Características locacionais } \\
\hline Água canalizada & $0,141^{\star *}$ & $0,066^{* * *}$ \\
\hline Escoadouro sanitário & 0,043 & $0,086^{* *}$ \\
\hline Proximidade ao esgoto & $-0,091$ & $-0,049^{* *}$ \\
\hline Material das paredes & $0,201^{* * *}$ & $-0,011$ \\
\hline Material da cobertura & 0,029 & $-0,026$ \\
\hline \multicolumn{3}{|l|}{ Características da alimentação } \\
\hline Qualidade da alimentação & $-0,026$ & $-0,009$ \\
\hline Quantidade de alimento & 0,058 & $0,039^{* *}$ \\
\hline \multicolumn{3}{|c|}{ Interações entre tipo de arranjo familiar e qualidade da alimentação } \\
\hline Monoparental fem. e qualidade da alimentação & $-0,045$ & 0,063 \\
\hline Monoparental masc. e qualidade da alimentação & $-0,412$ & $-0,246^{*}$ \\
\hline Outros arranjos e qualidade da alimentação & $-0,015$ & $0,203^{*}$ \\
\hline
\end{tabular}

Nota: Nível de significância: ${ }^{\star} 10 \%,{ }^{*} 5 \%,{ }^{* *} 1 \%$.

Fonte: Elaborado pelos autores a partir dos resultados da pesquisa.

lhos têm influência positiva sobre a saúde infantil quando comparado com crianças filhas de pais mais jovens. Isso porque há uma probabilidade maior de mães jovens ficarem mais expostas a fatores desfavoráveis durante a gestação e continuarem vivendo nas mesmas situações após o parto, o que resultaria em problemas para seu fi1 ho ${ }^{26,27}$. No que se refere à altura do responsável, ela também foi positiva e estatisticamente significativa tanto para o escore $\mathrm{z}$ da "altura para idade" quanto do "peso para altura", assinalando que a herança genética é um importante determinante dos índices antropométricos infantis.

Quanto à análise das características locacionais, observa-se que a presença no domicílio de água canalizada e algum tipo de escoadouro sanitário influenciou positivamente o escore $\mathrm{z}$ da "altura para idade" e do "peso para altura" das crianças. Já a proximidade ao esgoto teve efeito negativo sobre o escore z do "peso para altura". 
Além disso, se o material que predomina nas paredes dos domicílios era alvenaria houve um efeito positivo sobre o escore $\mathrm{z}$ da "altura para idade". Esses resultados indicam que crianças de domicílios com acesso aos serviços de saneamento básico e melhores condições de moradia tem menor probabilidade de desenvolverem diversos tipos de doenças e problemas nutricionais. Resultados análogos foram encontrados por Pimentel et al. ${ }^{28}$.

Sobre às variáveis de características da alimentação, quando a quantidade de alimento consumida no domicílio é sempre suficiente, há uma influência positiva sobre o escore $\mathrm{z}$ do "peso para altura". A variável "qualidade de alimentação" teve efeito negativo, porém não-significativo, sobre os índices antropométricos. Crianças com alimentação mais saudável e com acesso a quantidades suficientes de alimentos tendem a apresentar melhores indicadores de saúde. Para Garcia et al. ${ }^{29}$, melhorar a qualidade da alimentação tem sido uma das formas mais efetivas para manutenção da saúde e redução da mortalidade infantil. No Acre, crianças com deficiência de ferro foram as que apresentaram maior prevalência de déficit de altura para idade.

Em relação à influência do tipo arranjo domiciliar, apenas o arranjo monoparental feminino impactou de forma significativa e positiva o escore $\mathrm{z}$ da "altura para idade, quando comparado com a categoria base "Casal com filhos". Esse resultado indica que, controlando para os demais fatores como renda, escolaridade etc., em domicílios onde a mãe não tem a presença do cônjuge, as crianças apresentam-se com melhores indicadores de saúde do que em domicílios onde há a presença do cônjuge. Uma possível explicação pode ser a possibilidade de as mães passarem a dar maior atenção e terem mais tempo para dedicar aos filhos quando não há a presença do cônjuge. Resultados na mesma linha foram encontrados por Lansford ${ }^{30}$, em que as crianças de famílias monoparentais dos Estados Unidos desenvolveram-se sem prejuízos quando comparadas com crianças de famílias tradicionais. O estudo de Larson et al. ${ }^{31}$ para os Estados Unidos também apontou conclusões semelhantes. De acordo com Marin et al..$^{32}$, pesquisas têm apontado que mães solteiras estão, cada vez mais, procurando equilíbrio entre o trabalho e família, estando mais atentas ao cuidado à família. Assim, acredita-se que a ausência do pai/companheiro não traz necessariamente consequências negativas para as crianças desse arranjo.

Quanto às interações entre tipo de arranjo familiar e qualidade da alimentação, apenas a inte- ração entre qualidade da alimentação e o arranjo monoparental masculino teve efeito significativo e negativo, quando comparado com a categoria base "Casal com filho e qualidade da alimentação", indicando que ser do arranjo monoparental masculino e ter uma má alimentação tem influência negativa adicional sobre o escore $\mathrm{z}$ do "peso para altura" das crianças. Ressalta-se que para os dois índices antropométricos analisados, a interação entre qualidade da alimentação e arranjo monoparental masculino teve efeito negativo. Segundo Oliveira et al.$^{33}$, homens são geralmente menos preocupados com a saúde e apresentam menor conhecimento sobre as recomendações dietéticas atuais, o que pode explicar o efeito negativo dessa interação sobre as medidas antropométricas.

\section{Considerações finais}

Ao analisar a influência dos arranjos domiciliares e outras variáveis sobre as medidas antropométricas das crianças, observou-se que estar localizado no Norte e Nordeste afeta negativamente os escores z da "altura para idade" e do "peso para altura". As características domiciliares e locacionais mostraram-se importantes para explicar os índices de saúde infantil. Em relação ao tipo de arranjo familiar, os resultados indicaram que arranjos monoparentais femininos têm influência positiva sobre a altura para idade das crianças quando comparado ao arranjo casal com filhos. Esse resultado é importante e pode sinalizar que as mães passam a dar maior atenção e cuidado aos filhos quando não tem a presença do cônjuge.

Os resultados são de grande importância para a formulação e implementação de políticas públicas voltadas principalmente para transferências de renda e melhoria de programas alimentares, com o intuito de aumentar o bem-estar dos arranjos familiares, principalmente dos monoparentais femininos. Como as características da alimentação mostraram-se importantes para melhorar as medidas antropométricas infantis, são importantes políticas em segurança alimentar e nutricional, conscientizando a população sobre os problemas decorrentes de uma má alimentação, visando a proteção e o favorecimento à saúde e assim, reduzindo a taxa de mortalidade e as doenças relacionadas aos hábitos alimentares. Essas políticas também podem incentivar e reforçar a importância do aleitamento materno exclusivo até os seis meses de idade, como forma de prevenção de doenças e outros possíveis pro- 
blemas no futuro. Além disso, as características locacionais também foram fatores importantes sobre as medidas antropométricas, sendo, dessa forma, necessárias políticas que garantem o fornecimento dos serviços de saneamento básico, como forma de redução de doenças e proteção à saúde.

Ressalta-se o mérito do estudo ao trabalhar com dados da POF, por se tratar de uma amostra complexa com algumas dificuldades em seu manuseio e conter o grande número de informações. Destaca-se como uma das principais limitações a falta de informações mais precisas sobre os filhos, como por exemplo, se o filho é adotivo ou biológico. Uma sugestão para futuras pesquisas seria uma análise mais ampla sobre os fatores que afetam os índices antropométricos infantis, não só em famílias monoparentais femininas, como em todos os arranjos familiares e outras faixas etárias.

\section{Colaboradores}

Os autores trabalharam igualmente em todas as etapas da elaboração do artigo.

\section{Financiamento}

$\mathrm{O}$ autor $\mathrm{AB}$ Coelho agradece o apoio financeiro do Conselho Nacional de Desenvolvimento Científico e Tecnológico (CNPq), por meio de Bolsa de Produtividade em Pesquisa. 


\section{Referências}

1. Leone ET, Maia AG, Baltar PE. Mudanças na composição das famílias e impactos sobre a redução da pobreza no Brasil. Econom Soc 2010; 19(1):59-77.

2. Fontes MB, Wajnmam S, Guedes GR. Arranjos mono(bi)parentais e sua estrutura orçamentária. Rev Bras Econom Domestica 2016; 27(1):5-30.

3. Cavenaghi S, Alves JED. Mulheres chefes de família no Brasil: avanços e desafios. $32^{a}$ ed. Rio de Janeiro: Escola Nacional de Seguros; 2018.

4. Alves JED, Cavenaghi S. Tendências demográficas, dos domicílios e das famílias no Brasil. Aparte Incl Soc Debate 2012; 24:1-33.

5. Maia K, Devidé Júnior A, Souza SCI, Araújo FB. O papel das mulheres pobres brasileiras na estrutura familiar monoparental feminina: uma análise do ano 2012. Rev Econom 2015; 17(2):97-122.

6. Instituto Brasileiro de Geografia e Estatística (IBGE). Censo 2010. Famílias e Domicílios. Rio de Janeiro: IBGE; 2010.

7. Camargo CF, Curralero CRB, Licio EC, Mostafa J. Perfil socioeconômico dos beneficiários do Programa Bolsa Família: o que o cadastro único revela? In: Campello T, Neri MC, organizadores. Programa Bolsa Família: Uma década de inclusão e cidadania. Brasília: IPEA; 2013.

8. Santos JS, Costa COM, Nascimento Sobrinho CL, Silva MCM, Souza KEP, Melo BO. Perfil antropométrico e consumo alimentar de adolescentes de Teixeira de Freitas - Bahia. Rev Nutr 2005; 18(5):623-632.

9. Menezes RCE, Lira PIC, Leal VS, Oliveira JS, Santana SCS, Sequeira LAS, Rissin A, Batista Filho M. Determinantes do déficit estatural em menores de cinco anos no Estado de Pernambuco. Rev Saude Publica 2011; 46(6):1079-1987.

10. Haddad L, Hoddinott J. Women's income and boygirl anthropometric status in the Côte d'Ivoire. World Develop 1994; 22(4):543-553.

11. Magalhães EIS. Déficit estatural e fatores associados em crianças de 6 a 24 meses atendidas em unidades de saúde do sudoeste da Bahia. Cad Saude Colet 2016; 24(1):84-91.

12. Bergamaschi D, Adami FS. Perfil antropométrico de crianças e adolescentes. Rev Cien Saude 2015; 17(1):53-60.

13. Saldiva SRDM, Silva LFF, Saldiva PHN. Avaliação antropométrica e consumo alimentar em crianças menores de cinco anos residentes em um município da região do semiárido nordestino com cobertura parcial do programa bolsa família. Rev Nutr 2010; 23(2):221229.

14. Campos JM, Akutsu RCCA, Silva ICR, Oliveira KS, Monteiro R. Gênero, segurança alimentar e nutricional e vulnerabilidade: o Programa das Mulheres Mil em foco. Cien Saude Colet 2020; 25(4):1529-1537.

15. Greene WH. Econometric Analysis. $7^{\mathrm{a}}$ ed. Upper Saddle River: Prentice Hall; 2012.

16. Instituto Brasileiro de Geografia e Estatística (IBGE). Microdados da POF 2008-2009 (Pesquisa de Orçamentos Familiares). Rio de Janeiro: IBGE; 2010.
17. Instituto Brasileiro de Geografia e Estatística (IBGE). Pesquisa de Orçamentos Familiares 2008-2009: Despesas, rendimentos e condições de vida. Rio de Janeiro: IBGE; 2010.

18. Organización Mundial de la Salud (OMS). Curso de capacitación sobre la evaluación del crecimiento del niño: versión 1. Ginebra: OMS; 2006.

19. Pereira IFS, Andrade LMB, Spyrides MHC, Lyra CO. Estado nutricional de crianças menores de 5 anos no Brasil: evidência de polarização epidemiológica nutricional. Cien Saude Colet 2017; 22(10):3341-3352.

20. Rodrigues RSN, Cavalcanti AMTS, Silva TM. Diagnósticos de enfermagem em adolescentes com excesso de peso. Rev Rene 2013; 14(1):187-198.

21. Rissin A, Figueiroa JN, Benício MHD, Batista Filho M. Retardo estatural em menores de cinco anos: um estudo "baseline". Cien Saude Colet 2011; 16(10):40674076.

22. Souza AM, Pereira RA, Yokoo EM, Levy RB, Sichieri R. Alimentos mais consumidos no Brasil: Inquérito nacional de alimentação 2008-2009. Rev Saude Publica 2013; 47(1):190-199.

23. Instituto Brasileiro de Geografia e Estatística (IBGE). Tábua completa de mortalidade para o Brasil, 2015. Rio de Janeiro: IBGE; 2016.

24. Primo CC, Nunes BO, Lima EFA, Leite FMC, Pontes $\mathrm{MB}$, Brandão MAG. Quais os fatores que influenciam as mulheres na decisão de amamentar? Invest Educ Enferm 2016; 34(1):198-210.

25. Pereira IFS, Andrade LMB, Spyrides MHC, Lyra CO. Estado nutricional de menores de 5 anos de idade no Brasil: evidências da polarização epidemiológica nutricional. Cien Saude Colet 2017; 22(10):3341-3352.

26. Vieira MLF, Bicalho GG, Silva JLCP, Barros Filho AA. Crescimento e desenvolvimento de filhos de mães adolescentes no primeiro ano de vida. Rev Paul Pediatr 2007; 25(4):343-348

27. Kabubo-Mariaraa J, Ndenge GK, Mwabu DK. Determinants of children's nutritional status in Kenya: evidence from demographic and health surveys. J Afri Econom 2008; 18(3):363-387.

28. Pimentel PG, Sichieri R, Salles-Costa R. Insegurança alimentar, condições socioeconômicas e indicadores antropométricos em crianças da região metropolitana do Rio de Janeiro/Brasil. Rev Bras Estud Popul 2009; 26(2):283-294.

29. Garcia MT, Granado FS, Cardoso MA. Alimentação complementar e estado nutricional de crianças menores de dois anos atendidas no programa saúde da família em Acrelândia, Acre, Amazônia Ocidental Brasileira. Cad Saude Publica 2011; 27(2):305-316.

30. Lansford JE, Ceballo R, Abbey A, Atewart A. Does family structure matter? a comparison of adoptive, two-parent biological, single-mother, stepfather, and stepmother households. J Marriage Fam 2001; 63:840851.

31. Larson R, Dworkin J, Gillman S. Facilitating adolescents constructive use of time in one-parent families. Applied Develop Sci 2001; 15(3):143-157. 
32. Marin A, Piccinini CA. Famílias uniparentais: a mãe solteira na literatura. Psico 2009; 40(4):422-429.

33. Oliveira MS, Lacerda LNL, Santos LC, Lopes ACS, Câmara AMCS, Menzel HJK, Horta PM. Consumo de frutas e hortaliças e as condições de saúde de homens e mulheres atendidos na atenção primária à saúde. Cien Saude Colet 2015; 20(8):2313-2322.

Artigo apresentado em 17/02/2020

Aprovado em 05/10/2020

Versão final apresentada em 07/10/2020

Editores-chefes: Romeu Gomes, Antônio Augusto Moura da Silva 